Huybers, K. \& Jennings, P. (1957). J. gen. Microbiol. 17, 112-116

\title{
A Pure Preparation of the Staphylococcal Typing Bacteriophage 42D
}

\author{
By K. HUYBERS AND PAMELA JENNINGS \\ Department of Bacteriology, University of Melbourne, Australia
}

SUMMARY: The staphylococcal typing-phage $42 \mathrm{D}$ is propagated on bacteria which are lysogenic for the phage 1363 , and the latter phage is present as a contaminant in preparations of phage $42 \mathrm{D}$. This contamination can be avoided by propagating a pure clone of phage $42 \mathrm{D}$ on non-lysogenic organisms. Such nonlysogenic propagating strains can be isolated after the lysogenic propagating organisms have been irradiated heavily with ultraviolet radiation.

In testing the reactions of twenty-five staphylococcal phages Rountree (1949) found that many produced a small number of plaques on organisms other than those of homologous type. As most of these phages had been propagated on lysogenic bacteria, she suggested that the anomalous reactions might be due to contamination of the preparations with phage derived from the propagating organisms. Williams \& Rippon (1952) in their description of the techniques used to phage-type Staphylococcus aureus pointed out that preparations of the staphylococcal typing-phages might be contaminated in this fashion, for it is known (Rountree, 1949) that sixteen of the typing-phage propagating strains are lysogenic. It has recently been shown that preparations of the typing-phages 79 (Rippon, 1956) and $47 \mathrm{C}$ (Rountree, 1956) are in fact contaminated in this way. The following experiments show that preparations of typing-phage $42 \mathrm{D}$ are similarly contaminated.

By using non-lysogenic organisms which had appeared spontaneously on subculture of the lysogenic propagating strain Rountree (1956) obtained a pure preparation of the phage $47 \mathrm{C}$. In the following experiments, nonlysogenic organisms were similarly used to obtain a pure preparation of phage $42 \mathrm{D}$. These non-lysogenic organisms were obtained by irradiating the lysogenic propagating strain with a heavy dose of ultraviolet radiation, a technique first used by Lederberg \& Lederberg (1953) to obtain non-lysogenic strains of Escherichia coli K12.

\section{METHODS}

Phages and organisms. The typing phage $42 \mathrm{D}$ is propagated on Staphylococcus aureus 1363 . This organism is known to be lysogenic (Williams Smith, 1948; Rountree, 1949) and the phage it produces, which has not previously been named, is referred to here as phage 1363. The various strains of $S$. aureus 1363 described in this paper are referred to as follows: (a) 1363 (1363): $S$. aureus 1363 carrying the prophage $1363 ;(b) 1363(-)$ : $S$. aureus 1363, non-lysogenic; $(c) 1363(42 \mathrm{D}): S$. aureus 1363 carrying the prophage $42 \mathrm{D}$. 
This latter strain was isolated as a derivative of a doubly lysogenic strain 1363 (1363.42 D) in the course of other experiments.

Phage 42 D forms plaques on staphylococci 1363 (1363) and $1363(-)$, and phage 1363 forms plaques on staphylococci $1363(42 \mathrm{D})$ and $1363(-)$. In each case the efficiency of plating on the lysogenic and non-lyosgenic strain is the same.

Media. Bacto heart-infusion broth (Difco) was used as the basal medium, with $2 \%(\mathrm{w} / \mathrm{v})$ Bacto agar (Difco) added to form heart-infusion agar, and $1 \%(\mathrm{w} / \mathrm{v})$ agar added to form soft agar. The agar media were routinely reinforced with $2 \times 10^{-3} \mathrm{M}-\mathrm{Ca}\left(\mathrm{NO}_{3}\right)_{2} \cdot 4 \mathrm{H}_{2} \mathrm{O}$ and $2 \times 10^{-3} \mathrm{M}-\mathrm{MgSO}_{4} \cdot 7 \mathrm{H}_{2} \mathrm{O}$, except when $2 \times 10^{-2} \mathrm{M}$-sodium citrate was added to form citrate agar.

Phage propagation and titration. Phages were propagated by the plate technique described by Williams \& Rippon (1952) and titrated as described by these authors. Their notation was used to record the results of these titrations. Layer agar lawns as described by Adams (1950) were made where indicated in the text, by adding $0.1 \mathrm{ml}$. of a 4-6 hr. shaken culture of the appropriate staphylococcus to $2 \mathrm{ml}$. soft agar.

Production of the non-lysogenic staphylococcal strain $1363(-)$. The nonlysogenic strain was isolated after irradiating the lysogenic strain 1363 (1363) with a heavy dose of ultraviolet radiation. A sample (0.1 ml.) of a $4 \mathrm{hr}$. shaken culture of the lysogenic strain (c. $10^{8}$ organisms) was spread over the surface of a citrate agar plate and irradiated for $10 \mathrm{~min}$. at a distance of $50 \mathrm{~cm}$. from the centre of a $17 \mathrm{~W}$. Westinghouse Sterilamp. The plate was then incubated at $37^{\circ}$ until the surviving organisms had formed colonies. Citrate agar was used because non-lysogenic colonies on this medium are protected from infection with free phage. To identify the non-lysogenic colonies, the plate was replica-plated on to a layer agar lawn of indicator organisms (i.e. organisms sensitive to phage 1363). As described by Lederberg \& Lederberg (1953), each lysogenic colony on the replica is surrounded by a zone of lysis, whereas non-lysogenic colonies are not. The latter were picked from the original citrate agar plate and shown to be sensitive to phage 1363 .

We used staphylococcus 1363 (-) as the indicator organism in these experiments, since this strain was available, having been isolated by chance in the course of other work. Other staphylococci sensitive to 1363 phage might equally well have been used; two such strains are listed by Rountree (1949).

\section{RESULTS}

Presence of contaminating phage 1363 in stock preparation of phage $42 \mathrm{D}$

Phage 42D was propagated on staphylococcus 1363 (1363), and then titrated on this strain and on 1363 (42 D). The titrations of two preparations are shown in Table 1. The titration on the propagating strain shows the routine test dilution (the highest dilution giving confluent lysis) for each preparation, while the titration on $1363(42 \mathrm{D})$ shows the level of contaminating phage present. It can be seen that when the first preparation is used at its routine test dilution the contaminating phage will be present in sufficient concentration to cause 
Table 1. Test for purity of stock preparations of staphylococcal typing phage $42 D$

\begin{tabular}{|c|c|c|c|}
\hline & \multirow[b]{4}{*}{ Phage dilution } & \multicolumn{2}{|c|}{ Indicator staphylococcus } \\
\hline & & $1363(1363)$ & $1363(42 D)$ \\
\hline & & \multicolumn{2}{|c|}{ Lytic effect } \\
\hline & & For phage $42 \mathrm{D}$ & $\begin{array}{c}\text { For } \\
\text { contaminating } \\
\text { phage } 1363\end{array}$ \\
\hline Preparation A & $\begin{array}{l}\text { Undiluted } \\
10^{-1} \\
10^{-2} \\
10^{-3} \\
10^{-4} \\
10^{-5} \\
10^{-6}\end{array}$ & $\begin{array}{c}\text { Confluent lysis } \\
\text { Confluent lysis } \\
\text { Confluent lysis } \\
\text { Confluent lysis } \\
++ \\
+ \\
\pm\end{array}$ & $\begin{array}{c}\text { Confluent lysis } \\
++ \\
++ \\
+ \\
- \\
- \\
-\end{array}$ \\
\hline Preparation B & $\begin{array}{l}\text { Undiluted } \\
10^{-1} \\
10^{-2} \\
10^{-3} \\
10^{-4}\end{array}$ & $\begin{array}{c}\text { Confluent lysis } \\
\text { Confluent lysis } \\
\text { Confluent lysis } \\
++ \\
+\end{array}$ & $\begin{array}{l}++ \\
\pm \\
- \\
- \\
-\end{array}$ \\
\hline
\end{tabular}

a \pm reaction, and that when this preparation is used undiluted the contaminating phage will give a confluent lysis reaction. When the second preparation is used at its routine test dilution the contaminating phage is diluted out, but when this preparation is used undiluted the contaminating phage will give a ++ reaction.

\section{Production of the non-lysogenic staphylococcal strain $1363(-)$}

The combined results of two experiments are shown in Table 2. It can be seen that the yield of non-lysogenic clones was extremely low, only two such clones being detected in 2214 survivors from $c .6 \times 10^{-8}$ organisms.

Table 2. Recovery rate of non-lysogenic strains of Staphylococcus following heavy ultraviolet irradiation

$\begin{array}{ccccc}\begin{array}{c}\text { Staphylococcus } \\ \text { irradiated }\end{array} & \begin{array}{c}\text { No. of cocci } \\ \text { irradiated }\end{array} & \begin{array}{c}\text { No. of colonies } \\ \text { surviving }\end{array} & \overbrace{\text { Lysogenic }}^{\text {No. of colonies }} & \begin{array}{c}\text { Non-lysogenic } \\ 1363(1363)\end{array} \\ \text { c. } 6 \times 10^{8} & 2214 & 2212 & 2\end{array}$

Isolation and propagation of a pure strain of phage $42 \mathrm{D}$

Stock preparations of phage 42D are, as we have shown above, contaminated with phage 1363. From such a preparation a pure strain of phage $42 \mathrm{D}$ was obtained by single plaque isolation in the following way. First, the stock preparation was titrated in layered lawns of the non-lysogenic staphylococcal strain $1363(-)$. In this titration both phage $42 \mathrm{D}$ and the contaminating phage 1363 formed similar plaques but, as can be seen from Table 1, most of the phage in the dilutions higher than $10^{-3}$ is likely to be phage $42 \mathrm{D}$. An isolated plaque 
was therefore picked from the $10^{-6}$ dilution into $2 \mathrm{ml}$. broth. A loop of this suspension was spotted on to appropriate indicator staphylococci to confirm that this isolate was in fact phage $42 \mathrm{D}$ and the remainder of the suspension was carried through three single plaque isolations on the non-lysogenic staphylococcal strain. This pure strain of phage was propagated on the nonlysogenic staphylococci and then titrated on them and on staphylococcus 1363 (42 D). It can be seen from Table 3 that there was no contaminating phage present.

Table 3. Test for purity of pure phage $42 \mathrm{D}$ preparation

\begin{tabular}{|c|c|c|}
\hline \multirow[b]{2}{*}{ Phage dilution } & \multicolumn{2}{|c|}{ Indicator staphylococcus } \\
\hline & $1363(-)$ & $\begin{array}{c}1363(42 D) \\
\text { (for contaminating } \\
\text { phage 1363) }\end{array}$ \\
\hline Undiluted & Confluent lysis & - \\
\hline $10^{-1}$ & Confluent lysis & - \\
\hline $10^{-2}$ & Confluent lysis & - \\
\hline $10^{-3}$ & $+t$ & - \\
\hline $10^{-4}$ & ++ & - \\
\hline $10^{-5}$ & \pm & - \\
\hline
\end{tabular}

\section{DISCUSSION}

The results presented here show that stock preparations of the staphylococcal typing-phage $42 \mathrm{D}$ are contaminated with phage 1363 , and that it is possible to obtain a preparation free from such contamination by propagating a pure strain of phage $42 \mathrm{D}$ on artificially made non-lysogenic staphylococci.

As an alternative to this technique, a pure preparation of phage $42 \mathrm{D}$ can be obtained by inducing phage synthesis in staphylococcus $1363(42 \mathrm{D})$ by ultraviolet irradiation following the technique of Lwoff, Siminovitch \& Kjeldgaard (1950). We found that Bacto tryptose (Difco) in $2 \%(w / v)$ solution reinforced with $2 \times 10^{-3} \mathrm{M}-\mathrm{Ca}\left(\mathrm{NO}_{3}\right)_{2} \cdot 4 \mathrm{H}_{2} \mathrm{O}$ was a suitable medium for this purpose. Although staphylococcus 1363 (42D) is inducible, it is of interest to record that the strain 1363 (1363) is only partially inducible and that this difference in the inducibility of the two prophages is maintained in the doublylysogenic strain $1363(1363.42 \mathrm{D})$. This method of obtaining a pure preparation of phage $42 \mathrm{D}$ is however of no practical advantage since in order deliberately to synthesize the staphylococcal strain $1363(42 \mathrm{D})$, it would be necessary first to obtain both non-lysogenic organisms and a pure preparation of phage $42 \mathrm{D}$.

Serological techniques cannot be used to separate phage $42 \mathrm{D}$ from the contaminating phage $\mathbf{1 3 6 3}$, for the two phages are serologically related. For example, an antiserum against the pure phage $42 \mathrm{D}$ with a $K$ value (Adams, 1950 ) of 32 had a $K$ value of 17 against the phage 1363. It is of interest to note that although these two phages are serologically related, there was no evidence of cross-protection by the corresponding prophages.

Finally, we have assumed throughout that staphylococcus 1363 (1363) is carrying only one prophage. If it should prove that this organism is carrying 
more than one prophage, then it would be necessary to eliminate these prophages in succession in order to obtain a completely non-lysogenic propagating strain.

We wish to thank Dr Phyllis M. Rountree for supplying the typing phage and its propagating strain, Professor S. Rubbo for helpful advice, and the National Health and Medical Research Council, Canberra, for partial financial support.

\section{REFERENCES}

Adams, M. H. (1950). Methods of study of bacterial viruses. Meth. med. Res. 2, 1. Lederberg, E. M. \& Lederberg, J. (1953). Genetic studies of lysogenicity in Escherichia coli. Genetics, 38, 51.

Lwoff, A., Siminovitch, L. \& KJeldgaARd, N. (1950). Induction de la production des bactériophages chez une bactérie lysogène. Ann. Inst. Pasteur, 79, 815.

RIPPON, J. E. (1956). The classification of bacteriophages lysing staphylococci. J. Hyg., Camb. 54, 213.

Rountree, P. M. (1949). The phenomenon of lysogenicity in staphylococci. J. gen. Microbiol. 3, 153.

Rountree, P. M. (1956). Variations in a related series of staphylococcal bacteriophages. J. gen. Microbiol. 15, 266.

Williams, R. E. O. \& Rippon, J. E. (1952). Bacteriophage typing of Staphylococcus aureus. J. Hyg., Camb. 50, 320.

Williams Smith, H. (1948). Investigation on the typing of staphylococci by means of bacteriophage. 1. The origin and nature of lysogenic strains. J. Hyg., Camb. 46, 74 .

(Received 11 February 1957) 